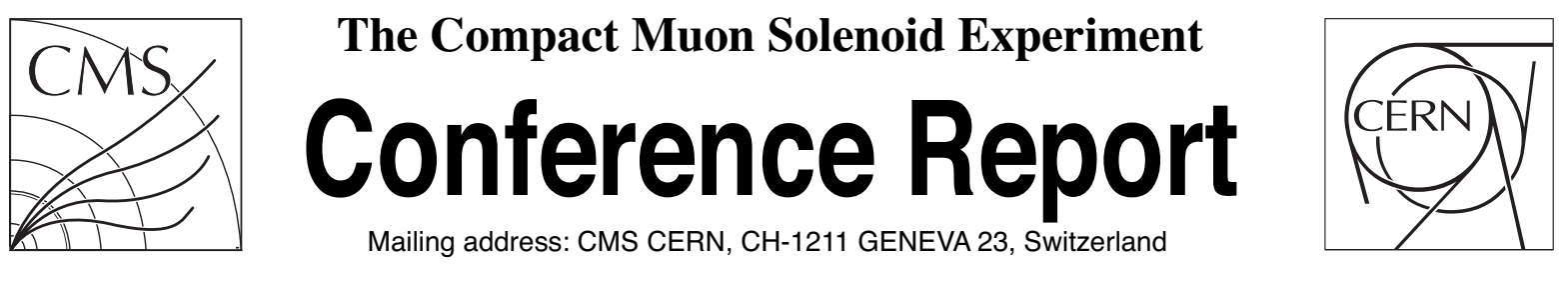

16 January 2012 (v3, 25 January 2012)

\title{
Future Silicon Sensors for the CMS Tracker Upgrade
}

\author{
Maria Bernard-Schwarz for the CMS Collaboration
}

\begin{abstract}
For the high-luminosity phase of LHC (Large Hadron Collider) at CERN a campaign was started in the CMS (Compact Muon Solenoid) experiment to investigate different radiation hard silicon detectors. Therefore 6" silicon wafers were ordered to answer various questions regarding for example the radiation tolerance and the annealing behavior of different sensor material. The testing variety includes sensor versions n-in-p and p-in-n in thicknesses from $50 \mu \mathrm{m}$ to $300 \mu \mathrm{m}$. In terms of sensor material the difference between floating zone, magnetic Czochralski and epitaxial grown silicon is investigated. For the n-in-p sensors, the different isolation technologies, p-stop and p-spray, are tested. The design of the wafer contains test structures, diodes, mini-sensors, long and very short strip sensors, real pixel sensors and double metal routing variants. The irradiation is done with mixed fluences of protons and neutrons which represent the rates of integrated hadrons that are expected in the CMS tracker after the LHC upgrade. This paper presents an overview of results from measurements of non-irradiated test structures with different technologies and also the results after irradiation.
\end{abstract}

Presented at HSTD8: 8th International "Hiroshima" Symposium on the Development and Application of Semiconductor Tracking Detectors 


\title{
Future Silicon Sensors for the CMS Tracker Upgrade
}

\author{
Maria Bernard-Schwarz ${ }^{1}$ on behalf of the CMS Tracker Collaboration \\ ${ }^{1}$ Institute of High Energy Physics \\ Austrian Academy of Sciences, Vienna, Austria \\ email: Maria.Bernard.Schwarz@assoc.oeaw.ac.at
}

\begin{abstract}
For the high-luminosity phase of LHC (Large Hadron Collider) at CERN a campaign was started in the CMS (Compact Muon Solenoid) experiment to investigate different radiation hard silicon detectors. Therefore 6" silicon wafers were ordered to answer various questions regarding for example the radiation tolerance and the annealing behavior of different sensor material. The testing variety includes sensor versions $n$-in-p and $p$-in- $n$ in thicknesses from $50 \mu m$ to $300 \mu \mathrm{m}$. In terms of sensor material the difference between floating zone, magnetic Czochralski and epitaxial grown silicon is investigated. For the n-in-p sensors, the different isolation technologies, p-stop and p-spray, are tested. The design of the wafer contains test structures, diodes, mini-sensors, long and very short strip sensors, real pixel sensors and double metal routing variants. The irradiation is done with mixed fluences of protons and neutrons which represent the rates of integrated hadrons that are expected in the CMS tracker after the LHC upgrade. This paper presents an overview of results from measurements of non-irradiated test structures with different technologies and also the results after irradiation.
\end{abstract}

Keywords:

CMS Tracker Upgrade, silicon sensors, process quality, irradiation, test structures PACS: 29.40.Gx, 29.40.Wk

\section{Motivation}

The LHC high luminosity upgrade will introduce an increase of the luminosity to $L=5 \cdot 10^{34} \mathrm{~cm}^{-2} \mathrm{~s}^{-1}$ by about 2022. This will lead to an expected fluence in the inner tracker layers of about $10^{16} n_{e q} \mathrm{~cm}^{-2}$, where $n_{e q}$ refers to the equivalent damage of $1 \mathrm{MeV}$ neutrons. The current CMS strip detector will reach the end of its lifetime after radiation damages will increase the full depletion voltage to the maximum voltage of the power supply. Furthermore, the higher the luminosity, the higher the track density and thus the occupancy of the detectors. One possible solution is to increase the granularity of the strips by using shorter strips. This introduces further challenges for the readout electronics as there are more channels to handle. There is also a need of a new powering and cooling system. The challenge for powering is to reduce power losses and heat dissipation of existing supply cables. In conjunction, cooling has to be more efficient with the constrains of reducing the mass of cooling pipes. Additionally, there will also be an earlier upgrade for the pixel tracker which will be implemented during the winter shutdown 2016/2017. A campaign within CMS has been initiated to define the sensitive parts of the new tracker after the LHC high luminosity upgrade.

\section{Testing Variety}

The campaign involves a collaboration of 17 research institutes, which started the investigations on radiation hard material and layout. In total, 158 silicon wafers were ordered from Hamamatsu Photonics K.K.; the design of each wafer can be found in [1]. Concerning the radiation hard material, three different silicon growth techniques are investigated. For the innermost part of the current CMS tracker a $320 \mu \mathrm{m}$ thick floating zone (FZ) material is used, whereas the outer parts use a $500 \mu \mathrm{m}$ thick floating zone material. Therefore this material serves as a reference and has also a high quality in terms of the high resistance values. The magnetic Czochralski (MCZ) wafer has a high oxygen 
concentration, and therefore is expected to be more radiation hard. The benefit of the epitaxial grown (Epi) silicon is the possibility to produce thin sensors. The variety covers thicknesses from $50 \mu m$ to $320 \mu m$ for $n$-in-p and p-in-n sensor types. For the n-in-p sensors, two different strip isolation technologies are tested. The sensor types named with letter P (p-stop) use a $p^{+}$layer between the $n^{+}$strips to intercept the electron accumulation layer, whereas sensor types labeled with the letter Y ( $\mathrm{p}$-spray) introduce a $\mathrm{p}$ doping on the full surface. The $\mathrm{p}$-in-n sensors are indicated with the letter $\mathrm{N}$. Wafers with a second metallization layer were also produced to investigate the radiation hardness and the noise behavior with directly connected electronics. Concerning the layout, the same wafer design is implemented on each wafer. There are standard baby sensors, which serve as a reference, diodes for material investigation and test structures for the determination of the process quality. The main focus of multigeometry pixel and strip structures are the capacitance measurements, as they lead to conclusions on the noise behavior. There are 12 different regions defined which use different strip pitches and different width-to-pitch ratios. One possible solution to cope with the higher track density would be the use of so called strixels, where the words strip and pixel are combined. These strixel sensors contain segmented strips with a larger area compared to the standard strip structures.

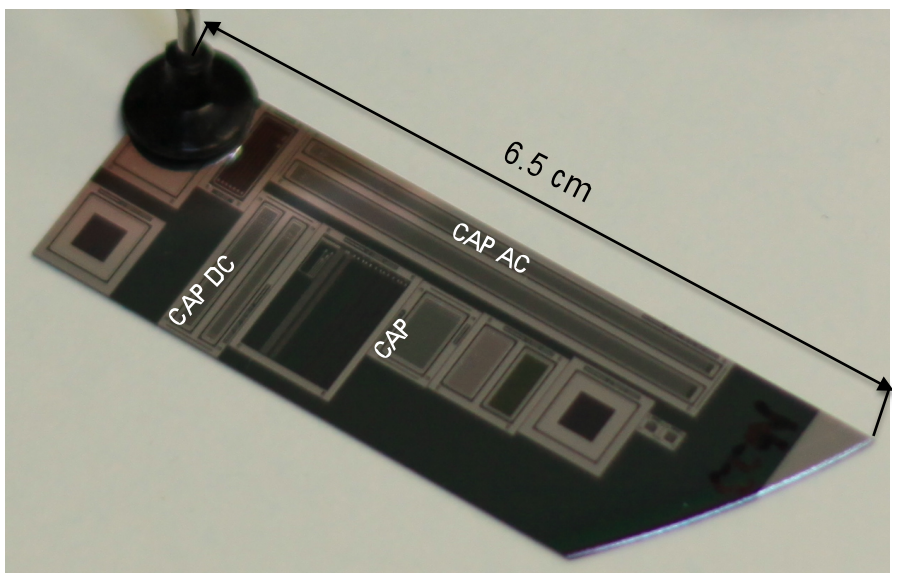

Figure 1: Picture of the CMS upgrade halfmoon.

In the following sections the results measured on the test structures are presented. The set of different test structures is called "halfmoon" within the community. A picture of the standard CMS upgrade halfmoon is seen in Fig.1. Ten different measurements can be performed with this halfmoon structure. The dark current of the detector is derived from the measurements on the diodes. The interstrip parameters are taken from test structures consisting of strips. For the interstrip capacitance measurement, the bias ring is connected to the strips with a polysilicon resistor. This structure is labeled with CAP AC. In contrast, the interstrip resistance is determined with the CAP DC structure. As a polysilicon resistance would distort the interstrip resistance measurement, the strips of this structure are isolated from the bias ring. The interstrip measurements are performed from the center strip to its two neighbors. The coupling capacitance and dielectric break down voltage are determined with the CAP structure where the strips are also isolated from the bias ring.

\section{Results for non-irradiated test structures}

To extract information on the process quality, different measurements on the halfmoon were performed. The results of the baby sensor measurements can be found in [1].

\subsection{Bulk dark current}

The bulk dark current is measured on diodes. Fig.2a shows several results for sensors of p type on floating zone material, where two samples of the thinnest and four samples of the thicker diodes have been measured. All types of the thinner floating zone materials showed an unexpected effect, as thinner diodes have a higher bulk dark current 
compared to the thicker ones. This effect is caused by defects which have been introduced during processing. The common method for producing thin sensors of floating zone material is wafer bonding, where a carrier wafer with low resistivity is bonded to the sensor material. A cost-saving method is deep diffusion where the active volume of the detector is less doped compared to the inactive volume. The differences in the processing can be verified with a spreading resistance profiling measurement [2]. The results are shown in Fig.2b. The gradient of the effective doping concentration resulting from the common method is steep, which is indicated by bold lines, in contrast to the measured profile of the diodes where deep diffusion has been applied. A deep hole trap was found as possible current generator, as reported in [3].

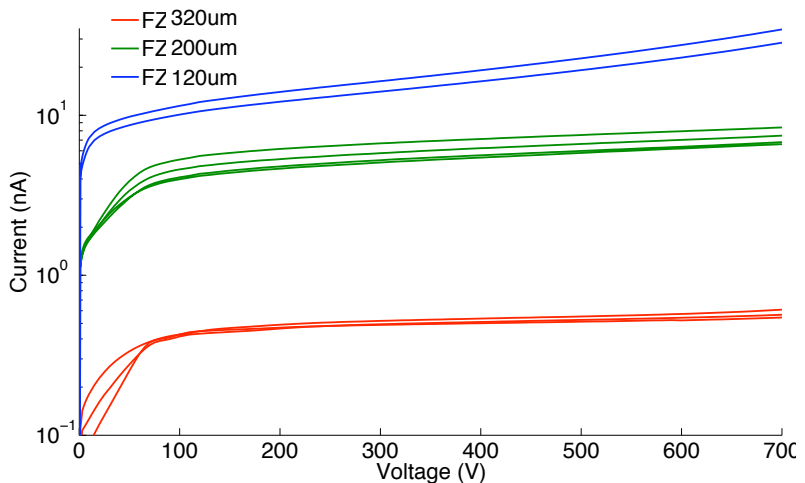

a)

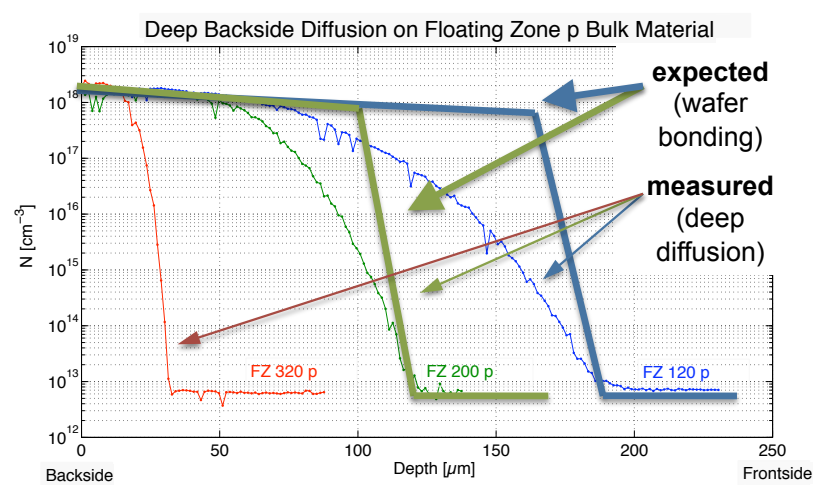

b)

Figure 2: a) Dark current and b) doping profile of floating zone diodes with three different thicknesses.

\subsection{Interstrip parameters}

The results of the interstrip capacitance show a distinct dependence on the substrate materials. The absolute capacitance values for the floating zone and magnetic Czochralski grown silicon are higher by a factor of 2 compared to those of the epitaxial grown material. The interstrip resistance for all materials is larger than $100 G \Omega$ with no significant differences related to material or thickness.

\subsection{Dielectric parameters}

The coupling capacitance and the dielectric break down voltage show no dependence on the different materials, as visible in Fig.3. Especially the break down voltage is remarkably homogenous. It is about $250 \mathrm{~V}$ for all materials. The electron microscopy shows that a thin layer of the dielectric oxide consists of a thermally grown oxide as it also extends into the bulk. The thicker part of the oxide has been grown by deposition. The dielectric layer is fabricated with a $280 \mathrm{~nm}$ thick $\mathrm{SiO}_{2}$ and a $50 \mathrm{~nm}$ thick $\mathrm{Si}_{3} \mathrm{~N}_{4}$ layer.

\section{Results for irradiated test structures}

The expected fluence, which will be reached after the high luminosity upgrade of LHC, has been calculated, and these numbers were used to plan the irradiation steps. The selected steps can be found in Table1 [4]. As the fluence depends on the radial distance from the interaction point, different irradiation steps are performed. For lower particle fluence the thicker materials are tested, whereas for higher particle fluence only the thinner materials will be irradiated. 

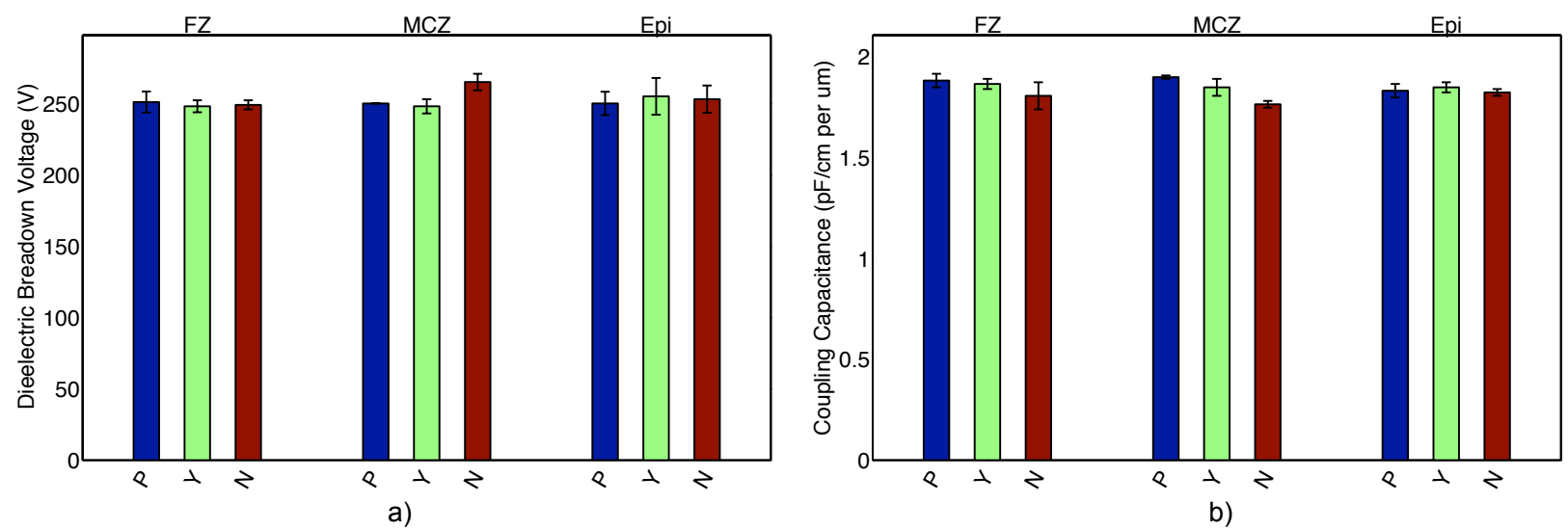

b)

Figure 3: a) Dielectric break down voltage and b) coupling capacitance of non-irradiated structures.

\begin{tabular}{|c|c|c|c|c|}
\hline radius & proton $\Phi_{e q}\left[\mathrm{~cm}^{-2}\right]$ & neutron $\Phi_{e q}\left[\mathrm{~cm}^{-2}\right]$ & total $\Phi_{e q}\left[\mathrm{~cm}^{-2}\right]$ & active thickness \\
\hline \hline $40 \mathrm{~cm}$ & $3 \cdot 10^{14}$ & $4 \cdot 10^{14}$ & $7 \cdot 10^{14}$ & $\geqslant 200 \mu \mathrm{m}$ \\
\hline $20 \mathrm{~cm}$ & $1 \cdot 10^{15}$ & $5 \cdot 10^{14}$ & $1.5 \cdot 10^{15}$ & $\geqslant 200 \mu \mathrm{m}$ \\
\hline $15 \mathrm{~cm}$ & $1.5 \cdot 10^{15}$ & $6 \cdot 10^{14}$ & $2.1 \cdot 10^{15}$ & $\geqslant 200 \mu \mathrm{m}$ \\
\hline $10 \mathrm{~cm}$ & $3 \cdot 10^{15}$ & $7 \cdot 10^{14}$ & $3.7 \cdot 10^{15}$ & $\leqslant 200 \mu \mathrm{m}$ \\
\hline $5 \mathrm{~cm}$ & $1.3 \cdot 10^{16}$ & $1 \cdot 10^{15}$ & $1.4 \cdot 10^{16}$ & $<200 \mu \mathrm{m}$ \\
\hline
\end{tabular}

Table 1: Overview of the irradiation campaign.

\subsection{Overview of the irradiation campaign}

The first irradiation step has already been carried out and different materials were irradiated with $1 \mathrm{MeV}$ neutrons from the reactor in Ljubljana and with $25 \mathrm{MeV}$ protons from the cyclotron in Karlsruhe. The particle fluence for the neutron irradiation was $4 \cdot 10^{14} n_{e q} \mathrm{~cm}^{-2}$, and it was $3 \cdot 10^{14} n_{e q} \mathrm{~cm}^{-2}$ for the proton irradiation. Short term annealing of 10 minutes at $60^{\circ} \mathrm{C}$ is performed after irradiation. Additional annealing effects introduced during the transport from the irradiation facilities to the individual institutes, where the measurements are performed, have to be considered. After these intermediate measurements, the mixed irradiation is continued where the originally proton-irradiated set will be neutron-irradiated and vice versa. For a complete statement on radiation hardness, full annealing studies with more annealing steps will be performed. For the dose measurements, a cross-check with the results of an irradiation at the reactor in Vienna has been done. The change of the bulk current $d I$ scaled with the volume of the structure $V$ can be compared by using $d I / V=\alpha \cdot \Phi_{e q}$ [5], where $\Phi_{e q}$ refers to the equivalent fluence of $1 \mathrm{MeV}$ neutrons and $\alpha$ to the current related damage rate. The dependency on annealing temperature and annealing time is taken into account with $\alpha$. The results from the irradiation in Vienna are in agreement with the dose measurements in Karlsruhe and Ljubljana.

\subsection{Short term annealing results}

There are no changes observed after irradiation for the interstrip capacitance and also no differences for the dielectric parameters. The decrease of the interstrip resistance can clearly be seen in Fig.4b. These values of the interstrip parameters are taken at $500 \mathrm{~V}$, where the structures are already full depleted. The strip isolation drops from several $100 G \Omega$ to the $M \Omega$ range. These measurements are in disagreement with the measurements performed on the baby sensors, where the value for isolation is about 10 to 100 times higher. In contrast to the test structure, the bias ring of the baby standard sensor is connected to the strips with a polysilicon resistor which has a lower resistance value compared to the interstrip resistance value. This causes a more complex measurement method compared to the simpler configuration on the test structure. Further investigations are needed to clarify the origin of this deviation. 


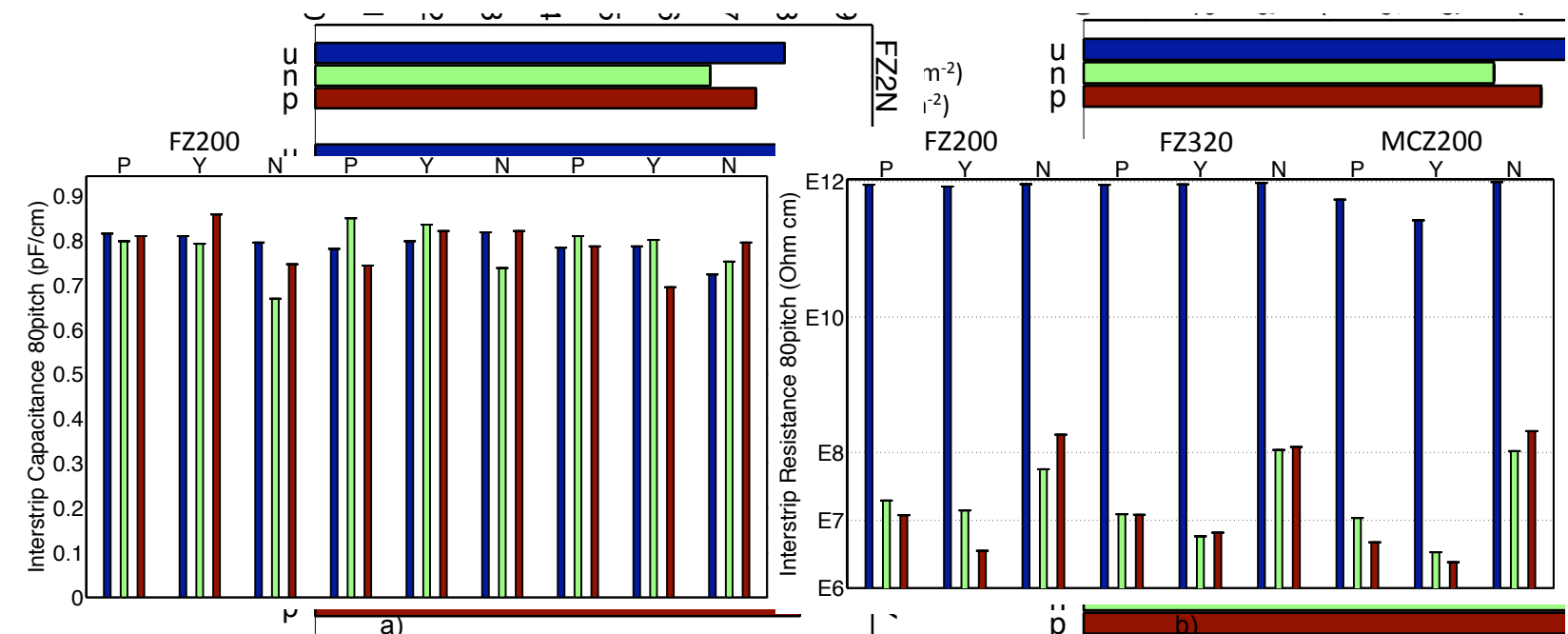

Figure 4: a) Interstrip capacitance and b) interstrip resistance after irradiation in comparison to the non-irradiated structures.

\section{Summary and Outlook}

Different detector technologies for the high luminosity upgrade of LHC are investigated. Most of the parameters before irradiation are in the expected range. The diodes with the deep diffusion process show an unexpected behavior in terms of the dark current. An additional order of the thinner floating zone materials, which are processed without the deep diffusion method, will be investigated. Only afterwards can the measurements before irradiation be concluded and the data concerning the process quality be summarized for all materials. The irradiation campaign has already started. The results of the short term annealing are not conclusive yet. Mixed irradiation of the first step and the following irradiation steps will be carried out soon. After this big irradiation campaign and the analysis has been completed, the sensitive parts of the new tracker can be defined.

\section{Acknowledgment}

I would like to thank the whole collaboration and also the AIDA project (Advanced European Infrastructures for Detectors at Accelerators) for funding the irradiation campaign. Many thanks to B. Lutzer and E. Fruehwirth, who did the measurements on irradiated structures, and W. Treberspurg for the electron microscopy studies and the doping profile measurements (all Institute of High Energy Physics, Vienna).

[1] A. Dierlamm, Silicon Sensor Developments for the CMS Tracker Upgrade, PSD9, Aberystwyth, 2011, CMS CR-2011/174

[2] W. Treberspurg, Material Analysis of Silicon Strip Sensors, Diploma Thesis, Vienna University of Technology, 2011

[3] A. Junkes, Influence of radiation induced defect clusters on silicon particle detectors, PhD thesis, Hamburg, 2011, DESY-THESIS-2011-031

[4] J. Erfle, Silicon Sensor Developments for the CMS Tracker Upgrade, RD11, Florence, 2011, CMS CR-2011/171

[5] M. Moll, Radiation Damage in Silicon Particle Detectors, Dissertation, University of Hamburg, 1999, desy-thesis-99-040 